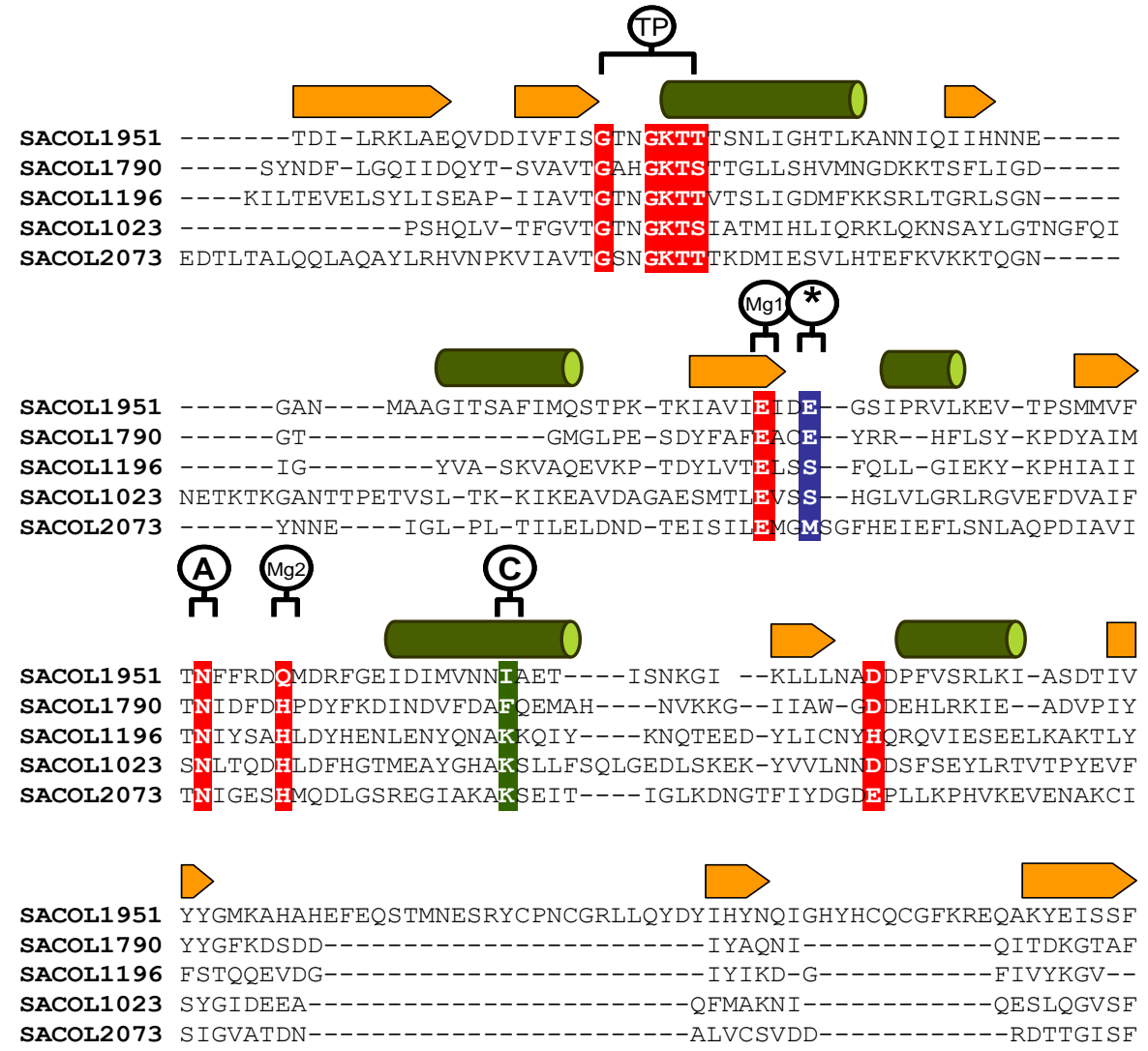

SACOL1951 TNFFRDQMDRFGEIDIMVNNIAET----ISNKGI --KLLLNADDPFVSRLKI-ASDTIV SACOL1790 TNIDFDHPDYFKDINDVFDAFQEMAH----NVKKG--I IAW-GDDEHLRKIE--ADVPIY SACOL1196 TNIYSAHLDYHENLENYQNAKKQIY----KNQTEED-YLICNYHQRQVIESEELKAKTLY SACOL1023 SNLTQDHLDFHGTMEAYGHAKSLLFSQLGEDLSKEK-YVVLNNDDSFSEYLRTVTPYEVF SACOL2073 TNIGESHMQDLGSREGIAKAKSEIT----IGLKDNGTFIYDGDEPLLKPHVKEVENAKCI

\title{
(A) A
}

SACOL1951 DVAPFLYLNI--NDEKYDMKIAGDFNAYNALAAYTVLRELGLNEQTIKNGFETYTSDNGR 298 SACOL1790 DV----YV-DGEFYDHFLSPQYGDHTVLNALAVIAISYLEKLDVTNIKEALETFGGVKRR 298 SACOL1196 ------RI INTEDLVL-----PGEHNLENI LAAVLA. I LAGVPI KA I I DSLTTFSGIEHR 311 SACOL1023 DF-----VTPFGTYPVKS-PYVGKENISNIMAAMIAVWSKGTSLETI IKAVENLEPVEGR 335 SACOL2073 T---------INNKEHYDLPI LGKHNMKNATI I I AVGHELGLTYNTI Y YNLKNVSLTGMR 318

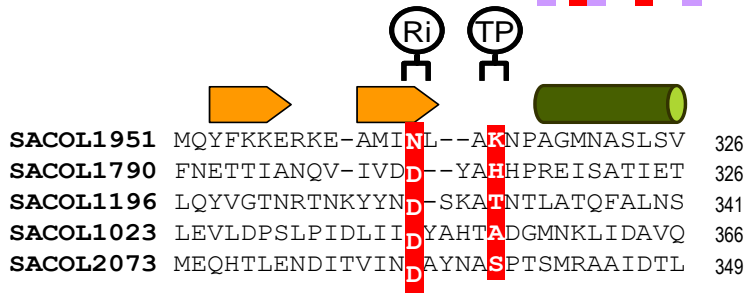


COBB BACME COBB_METJA COBB PSEDE Q5HEN̄2_STAAC

COBB_BACME COBB METJA COBB PSEDE Q5HEN2_STAAC

COBB BACME COBB METJA COBB PSEDE Q5HEN2_STAAC

COBB BACME COBB METJA COBB PSEDE Q5HEN2_STAAC

COBB_BACME COBB METJA COBB_PSEDE Q5HEN̄2_STAAC

COBB BACME COBB_METJA COBB PSEDE Q5HEN2_STAAC

COBB BACME COBB METJA $\mathrm{COBB}$-PSEDE Q5HEN2_STAAC

COBB_BACME COBB METJA COBB_PSEDE Q5HEN̄2_STAAC

COBB BACME COBB METJA $\mathrm{COBB}$ PSEDE Q5HEN2 STAAC
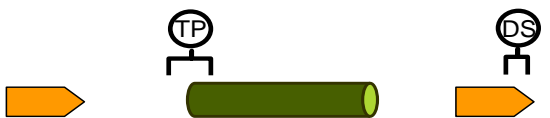

(1)

MSNRRLVIAGTGSGVPKTTLTIGLMAALKQAGYRVQGFKCGPDYIDPTYH MIMKRVVIAGTSSEVGKTVISTGIMKALSKK-YNVQGYKVGPDYIDPTYH MS--GLLIAAPASGS GKT TVTLGLMRALKRRGVAIAPGKAGPDYIDPAFH MH--ELTIYHFMS DKLNLYSDIGNI IALRQRAKK-RN------------

TAVTERTSRNIDSWMLEHDMVRE IVARASQDADIS IMEGVMGFFDGKNPI TIATGNKSRNLDSFFMNKEOIKYLFOKHSKDKDISVIEGVRGLYEGISAI AAATGEPCFNYDPWAMRPELLLANASHVASGGRTLIVEAMMGLHDG--AA

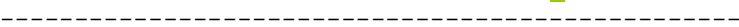

TNEGSTAEISLITNSPVLLVVNCASMARSAAAIVKGFOOFLPEANIVGVI DDIGSTASVAKALDSPI ILLVNAKSLTRSAIAI IKGFMSE-DNVKIKGVI DGSGTPADLAATLNLAVILVVDCARMSQSVAALVRGYADHRDDIRVVGVI ------------------------------------------- IKVNV-1

@ $\square$

R-GELTPFFEQLGQLVHDTIDIEKVYELALAPKIE INDPIFTKPS-VPQV NLKEIESKIVLWGELVEKYLDLDKIVEIADEDFEEVDDVFLWEVN-ENYK H-SALEGFIEAAAARVEAACDLDAIRLIATIF-PQVPAAADAERLRPLGQ

KIAVARDAAFNFYYEENFELLKACGAELVEFSPLKGEMVPQDADGLYIGG KIAVAYDKAFNFYYWDNFEALKENKAKIEFFSPLKDSEVP-DADILYIGG RIAVARDIAFAFCYEHLLYGWRQGGAEISFFSPLADEGPDAAADAVYLPG

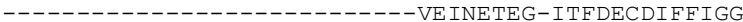

GFPEEFAETLAQQ IDVKNSVRAAIQKGLPTLAECGGFMFLTDGIVTTDDT GYPELFKEELSRNKEMI---ESIKEFDGYIYGECGGLMYITKS IDN---GYPELHAGQLSAAARFRSGMHSAAERGARI FGECGGYMVLGEGLVAADGT GSDREQALATKELSKIKTPLKEAIEDGMPGLTICGGYQFLGKKYITPDGT

CYEMVGLIPGQVRMQTKLAALGYREVT GKPGNFLFKGDIQAKGHEFHYST -VPMVGLLNCSAVMTKHVQGLSYVKAEFLEDCLIGRKGLKFKGHEFHYSK RYDMLGLLPLVTSFAERRRHLGYRRVVPV-DNAFFDGPM--TAHEFHYAT ELEGLGILDFYTESKTNRLT-G-DIVIE-SDT-FG--TI--VGFENHGGR
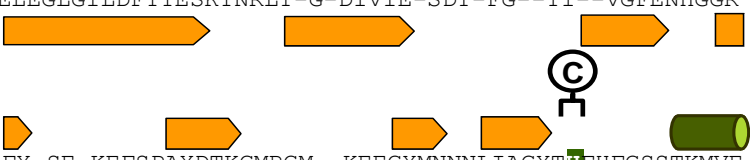

FY-SE-KEFSPAYDTKGMRGM--KEEGYMNNNI IAGYTHEHFGSSTKMVE LV-NI-KEERFAYKIERGRGI INNLDGI FNGKVLAGYLHNHAVANPYFAS IV-AE-GAADRLFAVSDAAGEDLGQAGLRRGPVAGSFMHLIDVA-----TYHDFGTLGHVTFGY--GNNDEDKKEGIHYKNLLGTYLHGP I LPKNYEIT 